REGARDS

SUR L'ECONOMIE ALLEMAND

BULLETIN ECONOMIQUE DU CIRAC
Regards sur l'économie allemande

Bulletin économique du CIRAC

115 | 2014

Varia

\title{
Le travail des seniors en Allemagne
}

\section{Karl Brenke}

Traducteur : Isabelle Bourgeois

\section{Q OpenEdition}

\section{Journals}

Édition électronique

URL : http://journals.openedition.org/rea/4762

DOI : $10.4000 /$ rea.4762

ISSN : 1965-0787

\section{Éditeur}

CIRAC

\section{Édition imprimée}

Date de publication : 1 décembre 2014

Pagination : 5-16

ISSN : 1156-8992

\section{Référence électronique}

Karl Brenke, «Le travail des seniors en Allemagne », Regards sur l'économie allemande [En ligne], 115 | décembre 2014, mis en ligne le 01 décembre 2016, consulté le 20 avril 2019. URL : http:// journals.openedition.org/rea/4762; DOI : 10.4000/rea.4762 


\section{Le travail des seniors en Allemagne}

\section{Karl Brenke}

En matière de travail des seniors, le gouvernement fédéral mène actuellement une politique à contre-courant de l'évolution réelle de la société et contraire aux intérêts économiques de l'Allemagne. Alors que, pour pérenniser le financement de l'assurance retraite, le processus pour porter progressivement, d'ici 2031, l'âge légal de départ à la retraite à 67 ans, venait tout juste de s'engager, la grande coalition a brusquement rebroussé chemin. Dorénavant, les actifs occupés ayant cotisé pendant 45 ans peuvent partir à la retraite dès l'âge de 63 ans sans que leur pension s'en trouve minorée.

Cette 'réforme' va à l'encontre d'une forte hausse - sans comparaison en Europe dans cette ampleur - du nombre d'actifs occupés de 65 ans et plus outreRhin. Si certains prolongent leur vie active par nécessité, la majorité d'entre eux sont mus par une motivation intrinsèque, c'est-à-dire par l'envie de rester en contact avec le monde professionnel. Dans une perspective économique aussi, une telle évolution est souhaitable. Car le vieillissement démographique fait que l'Allemagne ne peut se permettre de renoncer à une partie de sa main-d'œuvre qualifiée - et les seniors sont dans l'ensemble hautement qualifiés. Or si sont créées des incitations au départ anticipé à la retraite, une partie du capital humain si nécessaire se trouve littéralement mis en friche. Enfin, une telle politique n'apporte en rien plus de justice sociale. Car d'une part, elle stigmatise les seniors, laissant entendre qu'ils sont moins performants. Et d'autre part, elle fait peser de plus en plus lourdement le financement de l'assurance retraite légale sur les épaules des générations actuelles et futures de cotisants.

\section{Vieillissement démographique, mais taux d'activité en hausse chez tous les seniors}

La société allemande vieillit : à la fin 2013, la part des 60 ans et plus dépassait les $27 \%$, soit deux points de pourcentage de plus que dix ans auparavant. Le vieillissement démographique apparaît plus nettement encore quand on considère l'évolution de l'âge moyen : de 2001 à 2011 (dernières données disponibles), il est passé de 41,3 ans à 43,9 ans. Cette tendance va s'accélérer encore, l'espérance de vie s'allongeant du fait du progrès médical.

A quoi s'ajoute, dans le cas de l'Allemagne, un mouvement naturel de la population dont l'évolution s'effectue par vagues particulièrement prononcées, ainsi que le révèle la structure de la population résidente par âge (voir graphique page suivante).

Actuellement, la génération du baby-boom d'après-guerre arrive en nombre à l'âge de 60 ans. Cette vague massive atteindra son point culminant dans dix ans. Elle sera suivie d'un 'creux' avec l'arrivée de la génération, beaucoup moins nombreuse, née après l'introduction de la pilule contraceptive. Les cohortes nées ensuite ne cesseront de diminuer à leur tour. Quant à celles qui naissent actuellement, elles sont particulièrement peu nombreuses, représentant la moitié seulement de celles nées dans les années 1950 et jusqu'au début des années 1960.

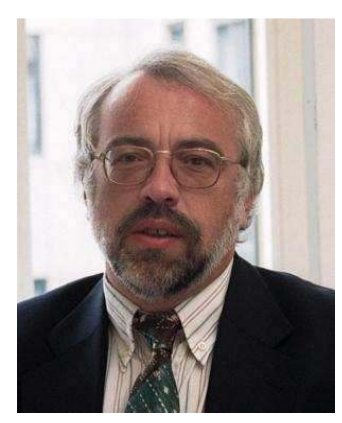

Karl Brenke, sociologue, spécialiste du marché du travail au DIW (Berlin)

Age moyen :

43,9 ans

Après la génération du baby-boom, celle de la pilule 


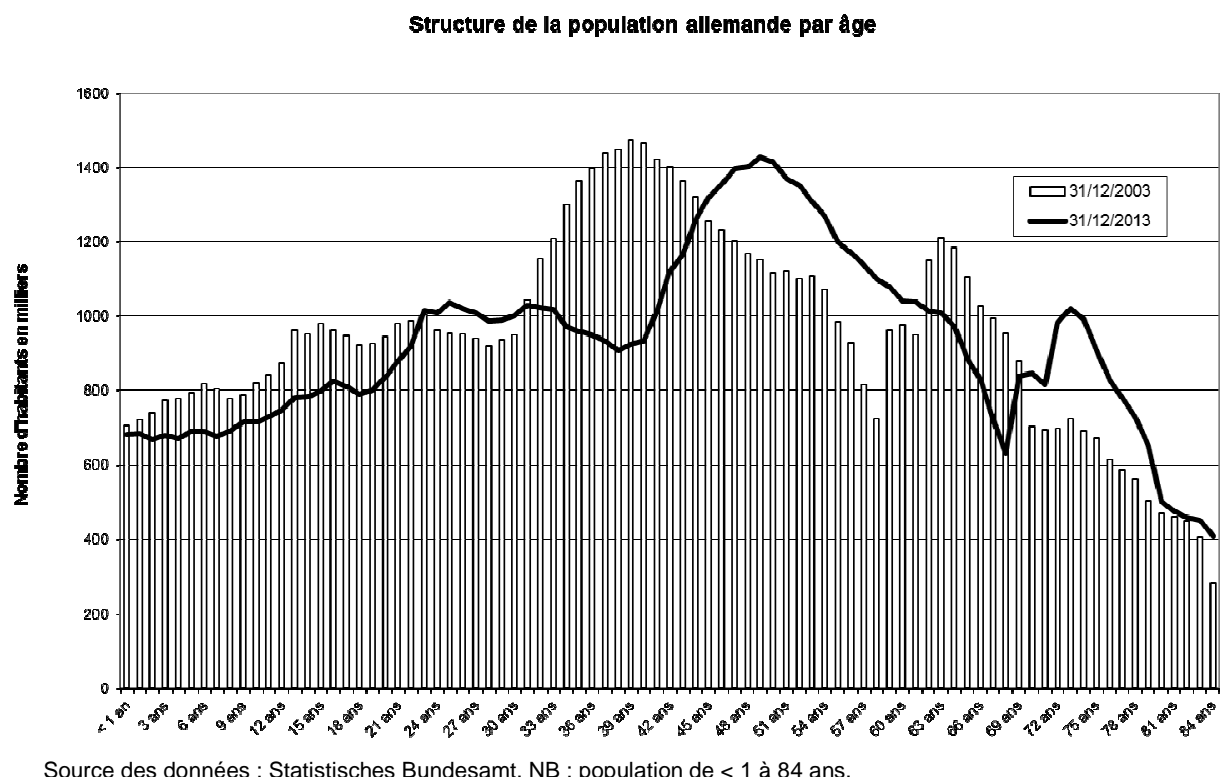

Pourtant, hausse du nombre d'actifs occupés

900000 plus de 64 ans dans la vie active
Normalement, une telle évolution de la population aurait dû se traduire déjà par une baisse du potentiel des actifs, c'est-à-dire des 15-64 ans selon les statistiques allemandes. Et de fait, la population dans cette tranche d'âge s'est réduite de 2,2 millions de personnes au cours des dix ans passés. Or au cours de la même décennie, le nombre de tous les actifs occupés, c'est-à-dire des personnes qui occupent ou recherchent un emploi rémunéré, a, lui, augmenté. SeIon les statistiques considérées, la hausse enregistrée varie entre 1,5 million (données macro-économiques de Destatis) et 2,8 millions de personnes (Enquête sur les Forces de Travail menée dans l'UE, EFT).

Cette différence dans l'évolution de la population en termes de démographie et d'activité trouve sa principale explication dans le profond changement observé dans la participation à la vie active : une part croissante de la population est en effet aujourd'hui intégrée dans le marché du travail. Alors qu'en 2003, la part des actifs occupés rapportée à l'ensemble de la population âgée de 15 à 64 ans (c'est-à-dire le taux de participation) était de $72,1 \%$, il s'établit à $77 \%$ dix ans plus tard. A cela, il faut ajouter toutefois que le périmètre traditionnel de la population en âge de travailler se trouve aujourd'hui décalé vers le haut dans la pyramide des âges. En effet, un nombre croissant des plus de 64 ans participe à la vie active : il est passé de 400000 en 2003 à 900000 dix ans plus tard.

Ces évolutions - modification de la participation et hausse du nombre d'actifs occupés - ne s'observent pas pareillement dans toutes les tranches d'âge (voir tableau 2). Ainsi, dans les plus jeunes, le taux d'activité n'a guère augmenté (effet de participation) entre 2003 et 2013 ; comme dans le même temps, leur part dans la population totale a légèrement diminué (effet démographique), le nombre des actifs occupés a quasi stagné. L'évolution dans les tranches d'âge moyennes est moins uniforme. Ainsi, le nombre d'actifs occupés de 35 à 44 ans a fortement baissé. Mais comme leur taux de participation, à un niveau élevé depuis toujours, n'a guère varié, ce recul en nombre a pour principale origine un facteur démographique : ceux qui, en vieillissant, sont sortis de cette tranche d'âge étaient plus nombreux que les plus jeunes entrant dans la même tranche. Ce vieillissement a fortement gonflé la tranche d'âge des 45 à 54 ans, caractérisée de surcroît par une forte hausse du taux de participation. Mais les tranches d'âge les plus décisives sont celles des 55 ans et plus. Au cours de la décennie écoulée, le taux d'activité y a en effet beaucoup augmenté, alors que le nombre de personnes dans ces cohortes a légèrement reculé. Cette évolution est particulièrement nette chez les 60-64 ans, et elle est considérable chez les plus de 64 ans. 


\section{Effet démographique et effet de participation}

Effet démographique : à partir du taux d'activité de l'année 2013 et du nombre d'habitants de 2013, on calcule le nombre d'actifs occupés hypothétiques qui résulterait d'un taux d'activité constant. La différence qui en découle avec le nombre d'actifs occupés recensés en 2003 est ce qu'on appelle l'effet démographique.

Effet de participation : pour le calculer, on soustrait l'effet démographique du nombre des actifs occupés recensés en 2003 et 2013.

Ces calculs prennent pour base les données du Mikrozensus (recensement partiel annuel effectué par l'Office fédéral des Statistiques), qui alimentent également l'Enquête sur les Forces de Travail menée dans l'UE.

\section{La plus forte hausse du travail des seniors en comparaison européenne}

Une participation accrue des seniors à la vie active s'observe dans presque tous les Etats de l'UE (sauf en Grèce, au Portugal, à Chypre et en Roumanie ; voir tableau 1). Cela dit, et exception faite des Pays-Bas, dans aucun Etat de I'UE, le taux d'activité des seniors n'a connu une aussi forte hausse qu'en Allemagne au cours de la décennie écoulée. Pour ce qui est du taux d'activité des 15-64 ans, l'Allemagne se situe dans la moitié supérieure en comparaison européenne. Plus généralement, elle n'est surclassée que par les Etats du nord de l'Europe, les pays anglo-saxons, ainsi que par la Suisse et les Pays-Bas.

Tableau 1 : Taux d'activité des seniors dans les pays européens

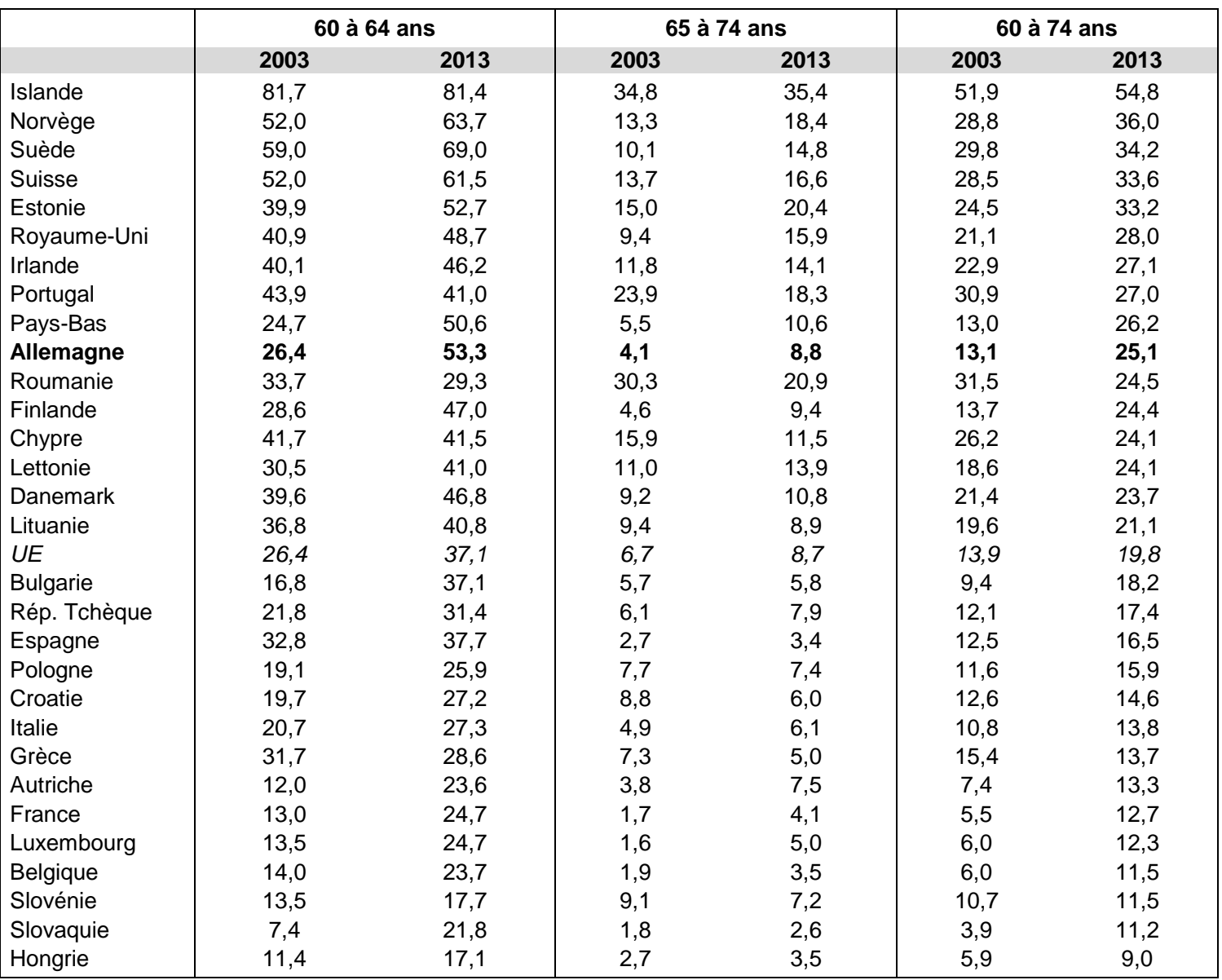

Source des données: Eurostat ; calculs propres à l'auteur.

En Allemagne, le taux de participation des 60-64 ans, donc des actifs s'approchant de l'âge de la retraite, a augmenté comme nulle part ailleurs. Cela place l'Allemagne en tête des Etats de l'UE, juste après la Suède ; seuls les Etats de I'AELE ont des taux plus élevés encore. II n'en reste pas moins que le taux d'activité des 60-64 ans représente plus du double de ce qu'il est par exemple en Pologne, en Autriche ou en France.
Taux d'activité des 60-64 ans en Allemagne : le double de ce qu'il est en France 
Reste à savoir comment s'explique le fait qu'en Allemagne, une part croissante des seniors participe à la vie active. Nous nous concentrerons ici particulièrement sur les 60 ans et plus - la définition usuelle allemande du senior sur le marché du travail. Après avoir examiné, sur la base de données empiriques, la composition de cette cohorte, nous analyserons les conditions cadre réservées à l'activité des seniors en Allemagne, et tout particulièrement celles de la transition entre la vie active et la retraite.

\section{Pas de différence entre les sexes}

Globalement, la participation à la vie active des femmes a plus fortement progressé que celle des hommes. Chez les femmes, elle a augmenté dans toutes les tranches d'âge. L'évolution du nombre d'actives occupées ne se distingue de celle des hommes que parmi les plus jeunes et dans les tranches d'âge moyennes où, en comparaison, le nombre d'actifs occupés a légèrement baissé chez les hommes. Parmi les plus âgés, il n'y a en revanche pas de différence nette entre les sexes : chez les hommes comme chez les femmes, le nombre d'actifs occupés a fortement augmenté en vertu de l'effet de participation. Etant donné que chez les seniors, la disparition du partage des rôles traditionnel ne suffit pas à expliquer la hausse de la participation, il faut en chercher les raisons ailleurs.

Tableau 2 : Evolution par tranche d'âge du nombre d'actifs occupés et du taux d'activité

\begin{tabular}{|c|c|c|c|c|c|}
\hline & \multicolumn{3}{|c|}{$\begin{array}{l}\text { Evolution du nombre d'actifs occupés } \\
\text { de } 2003 \text { à } 2013 \text { (en milliers) }\end{array}$} & \multicolumn{2}{|c|}{ Taux d'activité (en \%) } \\
\hline & Ensemble & $\begin{array}{l}\text { Effet démo- } \\
\text { graphique }\end{array}$ & $\begin{array}{c}\text { Effet de } \\
\text { participation }\end{array}$ & 2003 & 2013 \\
\hline & \multicolumn{5}{|c|}{ Ensemble } \\
\hline $15-24$ ans & 14 & -103 & 118 & 49,5 & 50,9 \\
\hline $25-34$ ans & 146 & -15 & 161 & 83,6 & 85,1 \\
\hline $35-44$ ans & -2431 & -2502 & 72 & 88,4 & 89,1 \\
\hline $45-54$ ans & 2091 & 1729 & 363 & 85,5 & 88,4 \\
\hline 55 - 59 ans & 1361 & 719 & 641 & 69,2 & 80,2 \\
\hline $60-64$ ans & 1204 & -208 & 1412 & 26,4 & 53,3 \\
\hline $65-69$ ans & 254 & -51 & 305 & 5,3 & 12,8 \\
\hline $70-74$ ans & 184 & 31 & 154 & 2,4 & 5,5 \\
\hline \multirow[t]{2}{*}{ Total } & 2824 & -401 & 3225 & 62,6 & 67,7 \\
\hline & \multicolumn{5}{|c|}{ Hommes } \\
\hline $15-24$ ans & 38 & 6 & 32 & 52,2 & 52,9 \\
\hline 25 - 34 ans & -54 & -45 & -9 & 90,6 & 90,4 \\
\hline $35-44$ ans & -1438 & -1403 & -36 & 95,7 & 95,0 \\
\hline $45-54$ ans & 1031 & 1033 & -2 & 92,7 & 92,7 \\
\hline $55-59$ ans & 573 & 377 & 196 & 78,9 & 85,8 \\
\hline $60-64$ ans & 518 & -150 & 668 & 35,2 & 61,7 \\
\hline $65-69$ ans & 161 & -29 & 191 & 6,6 & 16,3 \\
\hline $70-74$ ans & 113 & 23 & 90 & 3,6 & 7,5 \\
\hline \multirow[t]{2}{*}{ Total } & 942 & -188 & 1130 & 69,5 & 72,8 \\
\hline & \multicolumn{5}{|c|}{ Femmes } \\
\hline $15-24$ ans & -24 & -110 & 85 & 46,7 & 48,7 \\
\hline $25-34$ ans & 200 & 29 & 171 & 76,3 & 79,7 \\
\hline $35-44$ ans & -992 & -1100 & 108 & 80,8 & 82,9 \\
\hline $45-54$ ans & 1061 & 696 & 365 & 78,5 & 84,0 \\
\hline $55-59$ ans & 788 & 343 & 446 & 59,4 & 74,8 \\
\hline $60-64$ ans & 686 & -58 & 744 & 17,7 & 45,4 \\
\hline $65-69$ ans & 93 & -21 & 114 & 4,0 & 9,4 \\
\hline $70-74$ ans & 71 & 8 & 63 & 1,4 & 3,8 \\
\hline Total & 1883 & -212 & 2095 & 55,9 & 62,5 \\
\hline
\end{tabular}

Source des données : Eurostat, calculs de l'auteur.

L'une d'entre elles pourrait résider dans les modifications intervenues dans la structure des qualifications. En effet, en Allemagne comme dans les autres 
pays, le niveau de qualification est en hausse. Et celui ou celle qui a une bonne qualification a tendance à prolonger sa vie active jusqu'à un âge élevé, contrairement à celui ou celle qui n'a aucune formation professionnelle. II y a de multiples raisons à cela : les fonctions exigeant une bonne qualification sont plus intéressantes à exercer que les fonctions d'exécution et suscitent une motivation intrinsèque, alors que les tâches simples entraînent plutôt l'indifférence. En outre, ces dernières correspondent généralement à un travail manuel, c'est-àdire à un effort physique parfois pénible, ce qui fait que les actifs qui les occupent quittent souvent, pour des raisons de santé, la vie professionnelle prématurément, c'est-à-dire avant d'atteindre l'âge légal de départ à la retraite (voir Brussig, 2010). De plus, les perspectives de trouver un nouvel emploi ou de conserver l'emploi occupé varient grandement selon les qualifications.

\section{L'emploi des seniors a bénéficié de l'évolution favorable du marché du travail}

Ainsi, le taux d'activité est nettement plus élevé chez les actifs qualifiés que chez les moins qualifiés (voir tableau 3). De plus, le niveau de qualification de la population résidant en Allemagne a fortement augmenté - chez les plus jeunes comme chez les plus âgés. Quant à celui des 60-64 ans, il est le reflet de la politique conséquente de hausse des qualifications menée en Allemagne à l'époque où ils ont suivi leur formation professionnelle. Leur niveau de qualification est non seulement comparable en moyenne à celui des moins de 60 ans, mais il est également supérieur à celui des plus âgés, tout particulièrement de ceux qui ont atteint les 70 ans. Que, globalement, dans la tranche d'âge des 1569 ans, une part relativement élevée de la population soit sans formation professionnelle s'explique aisément: nombre d'actifs, et surtout les plus jeunes, sont en effet encore en formation.

Tableau 3 : Structure de la population et taux d'activité selon la qualification professionnelle

\begin{tabular}{|c|c|c|c|c|}
\hline & $15-59$ ans & $60-64$ ans & $65-69$ ans & $70-74$ ans \\
\hline & \multicolumn{4}{|c|}{ Structure de la qualification par tranche d'âge } \\
\hline & \multicolumn{4}{|c|}{2003} \\
\hline Pas de qualification ${ }^{1)}$ & 24,0 & 23,6 & 31,7 & 39,3 \\
\hline Qualification intermédiaire ${ }^{2)}$ & 55,6 & 56,1 & 51,9 & 45,9 \\
\hline Diplôme universitaire ${ }^{3)}$ & 20,4 & 20,3 & 16,4 & 14,8 \\
\hline \multirow[t]{2}{*}{ Total } & 100 & 100 & 100 & 100 \\
\hline & \multicolumn{4}{|c|}{2013} \\
\hline Pas de qualification ${ }^{1)}$ & 18,2 & 16,4 & 18,4 & 23,0 \\
\hline Qualification intermédiaire ${ }^{2)}$ & 56,8 & 57,7 & 57,6 & 55,4 \\
\hline Diplôme universitaire ${ }^{3)}$ & 25,1 & 25,9 & 24,0 & 21,6 \\
\hline \multirow[t]{2}{*}{ Total } & 100 & 100 & 100 & 100 \\
\hline & \multicolumn{4}{|c|}{ Taux d'activité } \\
\hline & \multicolumn{4}{|c|}{2003} \\
\hline Pas de qualification") & 54,4 & 17,8 & 4,2 & 1,4 \\
\hline Qualification intermédiaire ${ }^{2)}$ & 83,0 & 24,0 & 4,5 & 2,1 \\
\hline \multirow[t]{2}{*}{ Diplôme universitaire ${ }^{3)}$} & 92,4 & 44,3 & 10,4 & 6,4 \\
\hline & \multicolumn{4}{|c|}{2013} \\
\hline Pas de qualification ${ }^{1)}$ & 62,7 & 39,1 & 7,9 & 3,5 \\
\hline Qualification intermédiaire ${ }^{2)}$ & 84,5 & 51,0 & 11,4 & 4,9 \\
\hline Diplôme universitaire ${ }^{3)}$ & 92,2 & 67,3 & 19,9 & 9,5 \\
\hline
\end{tabular}

Source des données : Eurostat, calculs de l'auteur. a) ISCED 0 à 2 ; b) ISCED 3 à 4 ;c) ISCED 5 à 6 .

Du fait même que de plus en plus d'actifs qualifiés approchent de l'âge de la retraite, la participation des seniors à la vie active augmente donc mécaniquement. Mais cela n'explique pas tout, car le taux d'activité enregistre une forte progression indépendamment du niveau de qualification : aussi bien chez les détenteurs d'un diplôme universitaire que chez ceux qui disposent d'une quali- 
Taux de chômage des $60-64$ ans : $6,4 \%$

Un effet statistique aussi

Effet de l'affiliation selon les statuts professionnels fication intermédiaire (diplôme sanctionnant une formation professionnelle duale ou scolaire), et même chez ceux qui n'ont pas de formation professionnelle achevée. La hausse de l'emploi des seniors ne s'explique donc qu'en partie par l'évolution du niveau des qualifications.

Dans aucun autre pays de l'UE le taux de chômage n'est aussi bas qu'en Allemagne, et dans aucun autre pays, le chômage n'a aussi fortement reculé au cours des dix dernières années. Cela incite à penser que les conditions favorables sur le marché du travail ont joué un rôle non négligeable dans la hausse de l'activité des seniors. Un indicateur pourrait en être le recul du taux de chômage des actifs les plus âgés, ou plus exactement de ceux qui n'ont pas encore atteint l'âge légal de départ à la retraite. Et de fait, selon les statistiques de l'Enquête sur les Forces de Travail menée dans l'UE (EFT), le taux de chômage des 60-64 ans n'a cessé de baisser en tendance, tombant de 11,1\% en 2003 à $6,4 \%$ en 2013. Mais s'il a plus fortement reculé dans cette tranche d'âge que dans les autres, il n'en reste pas moins que les 60-64 ans sont plus souvent confrontés au sous-emploi que l'ensemble des plus jeunes, dont le taux se situait à 5,3 \% en 2013 (contre près de $10 \%$ en 2003).

Certes, les plus âgés se trouvent moins souvent au chômage que les plus jeunes (Bundesagentur für Arbeit, 2013), mais lorsqu'ils perdent leur emploi, leurs perspectives de réintégration sur le marché du travail sont faibles en comparaison, et le risque du chômage de longue durée est nettement plus élevé pour eux (Bundesagentur für Arbeit, 2014). Souvent, ces personnes perdent alors courage et se retirent mentalement de la vie active (l'enquête EFT ne les comptabilise dès lors plus comme chômeurs, puisque, comme ils abandonnent généralement la quête d'un emploi, ils ne sont plus à la disposition du marché du travail). Quoi qu'il en soit, l'amélioration des conditions d'accès à l'emploi a assurément contribué à la progression de l'activité des seniors.

II n'en a pas toujours été ainsi. Naguère encore, en période de chômage massif, nombre de mesures avaient été adoptées pour inciter les seniors au départ anticipé à la retraite dans le but de réduire le taux de chômage. La politique active pour l'emploi menée en Allemagne de l'Est juste après la chute du Mur en est un exemple patent ; les différentes mesures de retraite anticipée prises à ce moment-là avaient massivement retiré les actifs les plus âgés du marché de l'emploi : leur nombre a frisé par moments le million de personnes. De même, ce type de mesures visait et vise toujours à maquiller l'état réel du sous-emploi des seniors: aujourd'hui encore, les données administratives publiées par l'Agence fédérale pour l'emploi ne comptabilisent pas comme demandeurs d'emploi une partie des plus âgés, bien qu'ils le soient en réalité. En effet, lorsque, pendant un an environ, une personne de 59 ans et plus ne se voit pas proposer de place vacante par son agence locale pour l'emploi, elle n'est plus considérée comme chômeur et ne figure donc plus dans les statistiques. Si cette pratique reste monnaie courante, elle n'est toutefois plus aussi systématique que naguère.

\section{Nette hausse du nombre des actifs occupés ayant l’âge de la retraite}

Non seulement la participation à l'emploi des seniors a augmenté, mais également le taux d'activité effectif. II a de surcroît progressé plus vite que dans toutes les autres tranches d'âge et s'est tout particulièrement accru dans celle des plus âgés. Ainsi, chez les 70 ans et plus, le nombre des actifs occupant effectivement un emploi a presque triplé depuis 2003 et doublé chez les 65-69 ans - en partant, il est vrai, d'un niveau faible (tableau 4).

Cette évolution est d'autant plus surprenante que, jusqu'en 2013, l'âge légal de départ à la retraite se situait à 65 ans. En effet, les personnes de cet âge, puisqu'elles bénéficient des prestations de leur assurance retraite, n'ont par définition pas besoin d'exercer un emploi pour assurer leur subsistance. Or l'affiliation à une caisse de retraite légale n'est pas obligatoire dans tous les cas. Les 
fonctionnaires, par exemple, sont couverts par leur employeur (pouvoirs publics). Les personnes occupant un mini-job ne sont dans l'ensemble pas tenues de souscrire à une assurance retraite ; celle-ci n'est obligatoire (mais avec des taux de cotisation faibles) que pour les contrats de travail conclus depuis janvier 2013 et si la rémunération dépasse $400 €$ par mois (c'est-à-dire si elle est comprise entre 401 et $450 €)$. Quant aux travailleurs indépendants, ils peuvent, s'ils le désirent, souscrire à une assurance retraite ; mais cela n'est pas une obligation, ce qui explique la part importante, bien qu'en net recul, des travailleurs indépendants et des aides familiaux parmi les actifs occupés les plus âgés. Le nombre des indépendants âgés a certes augmenté au cours de la décennie écoulée, mais la hausse globale de l'activité des seniors est majoritairement imputable aux actifs occupant un emploi salarié. Chez les actifs occupés de moins de 60 ans, à l'inverse, on n'observe pas de telle modification de la structure de l'activité, la part des indépendants et des salariés ayant progressé de manière équivalente. En revanche, celle des aides familiaux a globalement perdu en importance.

Tableau 4 : Evolution des actifs occupés selon leur statut professionnel

\begin{tabular}{|c|c|c|c|c|}
\hline & Salariés & Indépendants & Aides familiaux & Total \\
\hline \multicolumn{5}{|l|}{$15-59$ ans } \\
\hline $\begin{array}{l}\text { Evol. } 2003 \text { à } 2013 \text { en \% } \\
\text { Part de tous les actifs occupés en \% } \\
\ldots 2003 \\
\text {.. } 2013 \\
\text { Nombre en } 2013 \text { en milliers }\end{array}$ & $\begin{array}{r}8,7 \\
89,7 \\
90,0 \\
33254,9\end{array}$ & $\begin{array}{c}9,6 \\
9,5 \\
9,7 \\
3569,8\end{array}$ & $\begin{array}{c}-58,2 \\
0,8 \\
0,3 \\
112,2\end{array}$ & $\begin{array}{c}8,2 \\
100 \\
100 \\
36936,9\end{array}$ \\
\hline \multicolumn{5}{|l|}{$60-64$ ans } \\
\hline $\begin{array}{l}\text { Evol. } 2003 \text { à } 2013 \text { en \% } \\
\text { Part de tous les actifs occupés en \% } \\
\ldots 2003 \\
\ldots .2013 \\
\text { Nombre en } 2013 \text { en milliers }\end{array}$ & $\begin{array}{c}105,1 \\
75,6 \\
83,4 \\
2169,3\end{array}$ & $\begin{array}{l}36,6 \\
21,6 \\
15,9 \\
412,8\end{array}$ & $\begin{array}{r}-52,6 \\
2,8 \\
0,7 \\
18,6\end{array}$ & $\begin{array}{l}85,9 \\
100 \\
100 \\
2600,7\end{array}$ \\
\hline \multicolumn{5}{|l|}{$65-69$ ans } \\
\hline $\begin{array}{l}\text { Evol. } 2003 \text { à } 2013 \text { en \% } \\
\text { Part de tous les actifs occupés en \% } \\
\ldots 2003 \\
\text {.. } 2013 \\
\text { Nombre en } 2013 \text { en milliers }\end{array}$ & $\begin{array}{r}123,9 \\
52,0 \\
59,6 \\
306,1\end{array}$ & $\begin{array}{l}84,1 \\
38,5 \\
36,3 \\
186,1\end{array}$ & $\begin{array}{r}-16,3 \\
9,5 \\
4,1 \\
21,0\end{array}$ & $\begin{array}{l}95,2 \\
100 \\
100 \\
513,2\end{array}$ \\
\hline \multicolumn{5}{|l|}{70 ans et plus } \\
\hline $\begin{array}{l}\text { Evol. } 2003 \text { à } 2013 \text { en \% } \\
\text { Part de tous les actifs occupés en \% } \\
\ldots 2003 \\
\text {... } 2013 \\
\text { Nombre en } 2013 \text { en milliers }\end{array}$ & $\begin{array}{r}276,8 \\
35,9 \\
47,6 \\
52,9\end{array}$ & $\begin{array}{l}48,1 \\
42,4 \\
55,7\end{array}$ & $\begin{array}{l}16,0 \\
10,0 \\
17,8\end{array}$ & $\begin{array}{l}184,1 \\
100 \\
100 \\
126,4\end{array}$ \\
\hline
\end{tabular}

Source des données : Eurostat, calculs de l'auteur.

Les actifs de 65 ans et plus exerçant un emploi salarié le font généralement à temps partiel (73\% en 2013). Le temps partiel est nettement moins développé chez les 60-64 ans (32\% seulement), et encore moins chez les personnes de 59 ans ou moins (un quart). L'âge légal de départ à la retraite trace ainsi une claire limite : une fois atteint ou dépassé, le temps plein n'est plus la règle.

\section{Si les retraités travaillent, ce n'est généralement pas pour vaincre la pauvreté}

En Allemagne, et en règle générale dans tous les pays industriels, les travaux sur la pauvreté comme les organisations luttant contre la pauvreté jouissent d'une grande attention dans l'espace public. Mais dans le cas des économies industrialisées, l'attention ne se focalise pas sur la pauvreté absolue, mais bien plutôt sur la pauvreté relative, c'est-à-dire sur la part de la population disposant 
Les retraités actifs sont loin d'être pauvres de revenus particulièrement faibles en comparaison du revenu médian relevé au sein d'un pays. Dès lors, on peut se demander si ce qui motive les retraités en Allemagne à exercer un emploi rémunéré à 65 ans passés n'est pas la nécessité d'apporter un complément de revenus au ménage pour compenser une trop faible pension de retraite.

Pour répondre à cette question, il convient de se pencher sur les données collectées en 2012 dans le cadre du Sozio-ökonomisches Panel (SOEP), une enquête annuelle menée, respectivement depuis 1984 dans l'ouest de l'Allemagne et depuis 1990 dans l'est, auprès d'un important panel représentatif des ménages (Wagner et al., 2008). On s'aperçoit ainsi que la durée hebdomadaire moyenne du temps de travail des 65 ans et plus se situe à 21,2 heures, alors qu'elle est de 37,7 heures chez tous les autres actifs occupés, qu'ils aient un emploi à temps plein ou à temps partiel. On s'aperçoit également que les revenus horaires (heures travaillées) issus du travail salarié (salaires et revenus des indépendants) sont légèrement inférieurs en brut chez les 65 ans et plus que chez les 15-64 ans lorsqu'il s'agit des revenus bruts, mais supérieurs quand il s'agit des revenus nets (voir tableau 5). Les revenus mensuels nets des 65 ans et plus ne sont en moyenne guère inférieurs à ceux de l'ensemble des 15-64 ans. Par contre, si on considère les revenus non pas par ménage mais par unité de consommation, c'est-à-dire si on tient compte du nombre et de l'âge des personnes composant le ménage, alors les revenus des 65 ans et plus sont nettement plus élevés que ceux des autres. Cela s'explique notamment par le fait qu'ils n'ont plus d'enfants à charge. En effet, pour calculer le revenu des unités de consommation, le revenu du ménage est divisé par la somme des facteurs attribués à ses différents membres: 1 à chaque adulte, 0,5 à chaque autre membre de 14 ans et plus, et 0,3 à chaque enfant de moins de 14 ans.

Tableau 5 : revenus moyens (en $€$ ) des actifs occupés de plus et de moins de 65 ans et des inactifs de 66 à 74 ans

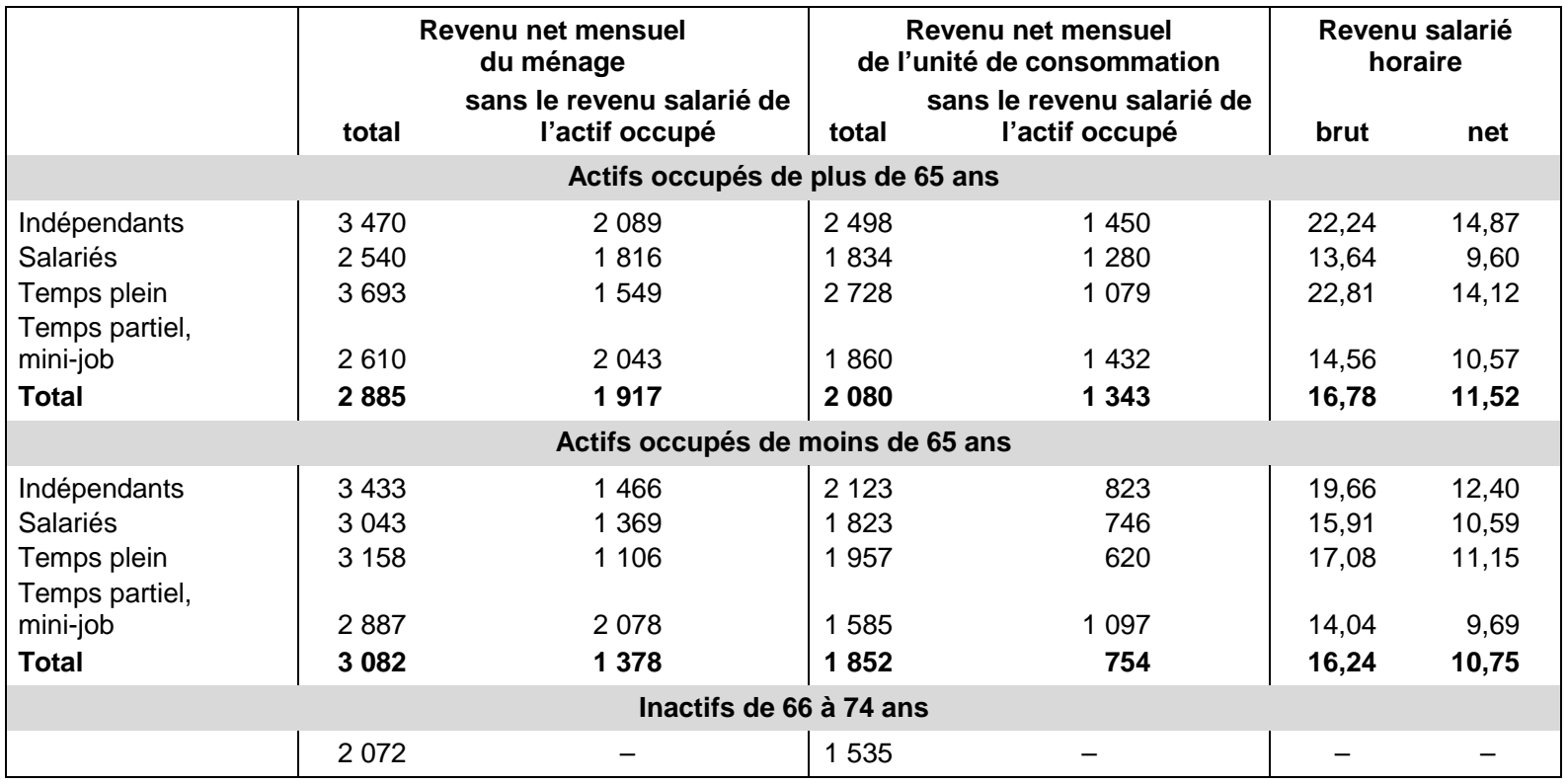

Source des données : Das sozio-ökonomische Panel (v28), calculs de l'Institut DIW de Berlin.

Or ce qui est déterminant pour comprendre pourquoi les seniors travaillent, c'est la question de savoir quel serait le niveau de revenu du ménage sans le revenu salarié du retraité. Sans ce dernier, le revenu net mensuel du ménage des actifs occupés de 65 ans et plus serait inférieur de près de $970 €$, celui de l'unité de consommation, de $740 €$. Mais même sans ce complément, le revenu du ménage des retraités actifs est tout sauf faible, s'établissant en moyenne à $1917 €$ (ou $1343 €$ par unité de consommation). Ce n'est que si le revenu net 
mensuel de ces unités de consommation se situait (hors complément) en-dessous de $1000 €$ qu'il friserait le seuil de pauvreté relative d'un foyer d'une personne.

Sans un revenu salarié complémentaire, un peu plus de $40 \%$ des actifs de 65 ans et plus se situeraient en-dessous du seuil de pauvreté relative (voir tableau 6). Cette part est particulièrement importante chez ceux qui occupent un emploi à temps plein ; leurs revenus sont inférieurs des deux tiers à ceux des indépendants. Moins d'un tiers seulement des seniors actifs a un revenu inférieur à $800 €$, ce qui correspond environ au seuil à partir duquel un célibataire perçoit des prestations sociales au titre des régimes d'assistance financés par l'impôt.

Autrement dit, s'il y a bien des seniors qui ont besoin de travailler au-delà de l'âge de la retraite pour accroître leurs revenus, la majorité des 65 ans et plus reste active par choix, et non par nécessité. Par ailleurs, nombre d'indépendants n'ayant que de faibles revenus (non salariés) seraient pourtant théoriquement en mesure de financer sans peine leur inactivité. Et s'ils choisissent de travailler au-delà de 65 ans, c'est probablement pour ne pas avoir à puiser dans leur épargne. Mais ce ne sont là que des hypothèses, impossibles à vérifier sur la base des données disponibles.
Un revenu complémentaire néanmoins bienvenu

Tableau 6 : Actifs occupés de plus de 65 ans percevant une pension de retraite et ayant de faibles revenus - part de tous les actifs occupés de plus de 65 ans (en \%)

\begin{tabular}{|c|c|c|c|c|}
\hline & $\begin{array}{r}\text { Personnes do } \\
\text { hor } \\
\text { est ir }\end{array}$ & $\begin{array}{l}\text { mensuel net } \\
\text { arié } \\
00 € €\end{array}$ & $\begin{array}{r}\text { Personnes do } \\
\text { hors } \\
\text { est } \mathrm{i}\end{array}$ & $\begin{array}{l}\text { mensuel net } \\
\text { arié } \\
\partial 0 €\end{array}$ \\
\hline & Unités & mation & & \\
\hline & $\geq 65$ ans & $\leq 64$ ans & $\geq 65$ ans & $\leq 64$ ans \\
\hline Temps plein & 62 & 77 & 54 & 67 \\
\hline mini-job & 34 & 52 & 23 & 37 \\
\hline Salariés & 39 & 70 & 30 & 58 \\
\hline Indépendants & 44 & 70 & 34 & 60 \\
\hline Total & 41 & 70 & 31 & 58 \\
\hline
\end{tabular}

Source des données : Das sozio-ökonomische Panel (v28), calculs de l'Institut DIW de Berlin.

Les données disponibles permettent ainsi d'affirmer que, dans la grande majorité des cas, ce sont des motifs intrinsèques et non pas la nécessité pécuniaire qui incitent les retraités actifs à travailler. Cela concorde aussi avec le fait que, bien que le contraire soit volontiers affirmé dans les débats publics, le phénomène des seniors pauvres n'est guère répandu en Allemagne (voir à ce sujet Grabka et al., 2012). Le désir de ne pas perdre son réseau social de contacts joue assurément un rôle majeur dans l'activité des seniors, car même s'ils n'occupent qu'un temps partiel, ils restent partie prenante dans la vie active et la société. Au total, on constate aussi que les retraités actifs sont plus heureux de leur vie que ceux qui ne travaillent plus (Brenke, 2013).

\section{L'impact décisif des réformes successives visant à préserver l'avenir du financement des retraites}

Le taux d'activité des plus âgés a fortement augmenté dans la plupart des Etats de l'UE, mais nettement plus en Allemagne qu'ailleurs. Cette hausse est imputable d'une part à l'évolution de la structure même de la population des actifs potentiels, dont le niveau de qualification s'est élevé ; en effet, les actifs hautement qualifiés restent plus longtemps actifs que les moins qualifiés. Dans le même temps, la structure des emplois a évolué elle aussi, se traduisant par un recul des tâches manuelles d'exécution, souvent physiquement pénibles.

En comparaison internationale, c'est en Allemagne que le taux d'activité des seniors a le plus augmenté, notamment sous l'effet de conditions de plus en
Les retraités restent actifs par choix de vie

Peu de pénibilité dans la structure des emplois

Bonnes perspectives d'embauche pour les seniors 
Une première hausse de l'âge légal de départ à la retraite décidée en 1992

Puis réduction des incitations au départ anticipé à la retraite

2007 : « retraite à 67 ans » - une mesure très controversée

Comment pérenniser au mieux le financement des retraites? plus favorables à l'emploi : le potentiel d'actifs se réduisant, les perspectives d'embauche augmentent. Ainsi, de 2003 à 2013, le nombre d'actifs a progressé de $13 \%$, trois fois plus qu'en moyenne européenne. C'est là une autre explication possible de la hausse généralisée de l'activité des seniors : elle s'observe en effet aussi bien chez les femmes que chez les hommes, aussi bien chez les mieux qualifiés que chez ceux qui n'ont aucune formation professionnelle.

Cependant, et bien qu'il soit impossible d'en chiffrer l'impact, il y a une troisième explication à la forte hausse de l'activité des seniors en Allemagne : elle réside dans l'évolution de la législation de l'assurance retraite. Durant les premières années après la Seconde Guerre mondiale, la politique allemande en matière de retraites avait surtout pour objectif de favoriser les retraités, plus particulièrement en aménageant leur départ anticipé à la retraite. Au fur et à mesure qu'on prenait conscience du fait qu'une telle politique risquait de mettre en danger le système de la retraite légale, un changement de cap s'est imposé. Ainsi, la réforme des retraites de 1992 prévoyait, outre une série de nouvelles dispositions, une hausse de l'âge légal de départ à la retraite - mais sa prise d'effet n'était programmée que pour 2001. D'autres Etats également avaient engagé des réformes de leurs systèmes de retraite dans les années 1990 pour préserver l'avenir de leur financement (voir Moog et al., 2011).

Au cours des années suivantes, et surtout au cours de la décennie écoulée, les réformes plus ou moins importantes du régime des retraites se sont succédé à un rythme quasi annuel en Allemagne. Parallèlement, un changement de paradigme s'opérait aussi en matière de politique pour l'emploi : on réduisit ainsi les incitations à un départ anticipé à la retraite dont l'objectif d'origine était réduire les tensions sur le marché de l'emploi. Ainsi, pour les seniors, la durée de versement des allocations chômage fut réduite à 18 mois sous le chancelier Schröder (avant d'être à nouveau portée à 24 mois par le gouvernement de grande coalition qui lui a succédé). Auparavant, cette durée était de 34 mois, et il n'était nullement fait obligation aux chômeurs indemnisés d'être disponibles pour la reprise d'un emploi. Cette disposition avait été massivement détournée pour anticiper un départ à la retraite : elle facilitait le licenciement des salariés les plus âgés à qui leur ancien employeur versait des indemnités cumulables avec les allocations chômage. Mais ce régime somme toute favorable à la situation individuelle des chômeurs seniors grevait lourdement le financement des systèmes de protection sociale.

En 2007 fut alors adoptée une réforme en profondeur du système des retraites, portant progressivement, de 2012 à 2031, l'âge légal de départ à la retraite à 67 ans. Cette mesure était vivement controversée dans l'espace politique - et elle l'est toujours : bien que cette réforme ait été adoptée par un gouvernement de grande coalition Union/SPD, le parti social-démocrate, puis Die Linke et le mouvement syndical n'ont cessé depuis de la remettre en question. Aujourd'hui, où le SPD fait à nouveau partie du gouvernement fédéral, bien qu'en tant que partenaire minoritaire, ce parti a imposé la possibilité d'un départ à la retraite à l'âge de 63 ans sans minoration des pensions. A condition toutefois que les prétendants aient cotisé pendant 45 ans (et que les périodes de chômage passées n'excèdent pas sept ans au total). Or seule une minorité remplit ces conditions : le ministère fédéral du Travail estime ainsi qu'un peu plus du quart seulement des personnes de 63 ans a accumulé 45 ans de cotisations.

La « retraite à 67 ans » est critiquée au motif qu'elle serait socialement injuste. Mais pour pérenniser le financement de l'assurance retraite, il n'y a que très peu de solutions. La première est de hausser l'âge légal de départ à la retraite, comme ce fut le cas de la réforme de 2007. Certes, une telle mesure peut être ressentie comme pénalisant ceux qui étaient à la veille de partir à la retraite selon l'ancien régime. D'un autre côté, il ne faut pas perdre de vue le fait que l'espérance de vie moyenne ne cesse d'augmenter, ce qui rallonge d'autant la période pendant laquelle les pensions sont versées aux retraités. Actuellement, une femme de 60 ans a une espérance de vie supérieure de un an à ce qu'elle 
était voici huit ans seulement, pour un homme de 60 ans, elle est même supérieure de un an et demi (Statistisches Bundesamt, 2012). II ne peut donc être question d'un quelconque préjudice lorsque l'âge légal de départ à la retraite est porté progressivement de 65 à 67 ans sur une durée de 19 ans. Cette mesure tient simplement compte de l'augmentation de l'espérance de vie et, partant, de l'augmentation de la durée pendant laquelle est perçue la pension.

Une autre mesure possible serait d'augmenter les cotisations à l'assurance retraite. Or, avec actuellement $18,9 \%$ du salaire soumis à cotisations sociales, les cotisations sont déjà à un niveau très élevé. La réforme des retraites de 2007 avait limité la hausse du taux de cotisations à $22 \%$ à longue échéance. Certes, arithmétiquement, il est toujours possible d'augmenter le taux de cotisations sur une courte période. Mais sous l'effet du vieillissement démographique, il ne serait plus possible, et ce à brève échéance, de respecter ce plafond de $22 \%$. Et une telle mesure porterait immédiatement préjudice aux actifs occupant un emploi soumis à cotisations sociales. Ce sont eux dès lors qui se sentiraient pénalisés par une telle hausse des cotisations et la ressentiraient comme socialement injuste. Les employeurs en pâtiraient eux aussi, puisque le financement de l'assurance retraite est assuré de manière paritaire. On peut donc en parfaite logique affirmer que la génération actuelle des retraités a un sort beaucoup plus enviable que celui de toutes celles à venir. En ce sens, il existe bel et bien une injustice de traitement - intergénérationnelle ; elle se trouverait démultipliée dans l'hypothèse d'une hausse des cotisations pesant sur les actifs occupés.

Une troisième solution pourrait consister dans la baisse du montant des pensions versées. Mais elle porterait préjudice à tous ceux qui perçoivent une pension de retraite ou sont à la veille d'en percevoir une. Et aucun gouvernement ne se résoudrait à prendre une telle mesure car il risquerait de ne pas être réélu. Quel que soit l'angle sous lequel on aborde le problème du financement de l'avenir des retraites, la hausse de l'âge légal de départ à la retraite reste la seule solution envisageable et la moins socialement injuste à la fois pour les retraités et pour ceux qui cotisent.

Théoriquement, il serait également possible de renflouer les caisses d'assurance retraite mises à mal par le vieillissement démographique en multipliant les dotations publiques prélevées sur l'encours fiscal. Mais cette solution serait socialement injuste, puisqu'elle mettrait à contribution même ceux qui ne bénéficieront jamais d'une prestation de l'assurance retraite légale, puisqu'ils n'y cotisent pas étant donné qu'un régime différent (privé) de protection vieillesse s'applique à eux, ce qui est notamment le cas des indépendants. En outre, cette solution présenterait l'inconvénient d'augmenter les dépenses publiques, un effet qu'il faudrait compenser soit par une hausse des prélèvements fiscaux alors même que ceux-ci sont déjà trop élevés, soit par une compression des dépenses publiques dans d'autres domaines sous peine de creuser encore la dette publique.

TOUTES CES QUESTIONS RENVOIENT, AU FOND, A DES CHOIX en matière de politique de redistribution. Or il faut se demander aussi quel est le choix le plus judicieux sous l'angle économique et social. Lorsqu'il existe des incitations à mettre une fin précoce à la vie active, elles sont tout naturellement saisies. Mais elles mènent aussi à des excès, en l'occurrence à l'exclusion des salariés les plus âgés. Jusqu'à il y a une dizaine d'années, les entreprises allemandes étaient littéralement en proie au "jeunisme »: seule des équipes les plus jeunes possibles étaient considérées comme efficientes, les salariés les plus âgés avaient la réputation d'être moins productifs. Or pour la société dans son ensemble, la mise à la retraite précoce de personnes tant mentalement que physiquement performantes se solde par une perte de compétitivité, puisqu'une partie du capital humain se trouve ainsi comme mise en friche. Et que dire, enfin, du respect de soi d'un senior lorsqu'on lui fait sentir qu'on n'a plus besoin de lui ?
Une hausse des cotisations serait contraire à la solidarité intergénérationnelle

Une baisse du montant des pensions serait socialement injuste

Des dotations publiques creuseraient encore plus la dette 
Depuis, les mentalités ont commencé à changer. Nombreux sont aujourd'hui les employeurs qui savent apprécier à leur juste valeur la qualification et les compétences spécifiques d'un salarié senior comme l'expérience, la fiabilité ou la loyauté. Pourtant, des progrès restent à faire : il n'y a pas encore assez de modèles de temps de travail ou de programmes de santé internes aux entreprises adaptés aux plus anciens, et un effort accru visant à les impliquer dans la formation continue s'impose. Mais le plus important reste la motivation des seniors. Car quand on donne à ses collaborateurs l'impression qu'ils sont devenus inutiles parce que leurs performances seraient insuffisantes, on les pousse à l'émigration intérieure, générant ainsi un engrenage de prophétie autoréalisatrice.

Ce changement de mentalité doit gagner en profondeur en Allemagne car du fait du vieillissement des actifs potentiels, les employeurs devront à l'avenir miser de plus en plus sur les seniors pour préserver leur compétitivité. A l'inverse, toute politique qui, sous couvert de « justice sociale », multiplie les incitations au départ anticipé à la retraite ne peut être que contreproductive pour l'économie. Sans compter qu'elle n'a rien de socialement juste non plus.

Traduction : I. Bourgeois

\section{Indications bibliographiques}

- BRENKE K., «Immer mehr Menschen im Rentenalter sind erwerbstätig », Wochenbericht des DIW, n०6/2013

- Brussig M., Erwerbstätigkeit im Alter hängt vom Beruf ab. Ausdifferenzierung der Erwerbschancen vor allem nach dem 60. Lebensjahr, in einigen Berufen aber schon früher. Altersübergangsreport, $\mathrm{n}^{\circ} 5 / 2010$

- Bundesagentur für ARBeIt, Arbeitsmarktberichterstattung: Der Arbeitsmarkt in Deutschland. Ältere am Arbeitsmarkt, Nuremberg, 2013

- Bundesagentur für ARBeIt, Arbeitsmarktberichterstattung: Der Arbeitsmarkt in Deutschland. Die Arbeitsmarktsituation langzeitarbeitsloser Menschen, Nuremberg, 2014

- GrabKa M., GöBel J., SchupP J., «Höhepunkt der Einkommensungleichheit in Deutschland überschritten ? ", Wochenbericht des DIW, n43/2012

- Moog S., MüLLER C., «Zur Erhöhung der Altersregelgrenze in Deutschland - eine internationale Perspektive ", Vierteljahrshefte zur Wirtschaftsforschung, n²/2011

- Statistisches Bundesamt, Periodensterbetafeln für Deutschland, Wiesbaden, 2012

- Wagner G. G., Göbel J., Krause P., Pischner R., Sieber I., « Das Sozio-oekonomische Panel (SOEP) : Multidisziplinäres Haushaltspanel und Kohortenstudie für Deutschland », AStA Wirtschafts- und Sozialstatistisches Archiv 2, 2008, $\mathrm{n}^{\circ} 4$. 